\title{
CircCDC45 promotes the malignant progression of glioblastoma by modulating the miR-485-5p/CSF-1 axis
}

\author{
Rongcai Liu, Weimin Dai* $\mathbb{D}^{*}$ An Wu and Yunping Li
}

\begin{abstract}
Background: Glioblastoma (GBM) is characterized by progressive growth and metastasis. Numerous studies claim that the deregulation of circular RNAs (circRNAs) is associated with cancer progression. However, the role of circRNAs in GBM is largely limited. The purpose of this study was to investigate the functions of circCDC45 in GBM and provide a feasible functional mechanism to support its role.

Methods: The expression of circCDC45, miR-485-5p and colony-stimulating factor 1 (CSF-1) mRNA was examined using quantitative real-time polymerase chain reaction (qRT-PCR). Cell proliferation was assessed using cell counting kit -8 (CCK-8) assay and colony formation assay. Cell migration and cell invasion were monitored using transwell assay. The protein levels of proliferation-related markers and CSF-1 were determined using western blot. The target relationship was predicted using bioinformatics tools and validated using dual-luciferase reporter assay and RNA immunoprecipitation (RIP) assay. Animal models were constructed to verify the role of circCDC45 in vivo.

Results: The expression of circCDC45 and CSF-1 was elevated in GBM tissues and cells, while the expression of miR-485-5p was declined. Downregulation of circCDC45 or CSF-1 blocked GBM cell proliferation, invasion and migration as well as tumor growth in vivo. In mechanism, circCDC45 positively regulated the expression of CSF-1 by targeting miR-485-5p. Inhibition of miR-485-5p reversed the biological effects caused by circCDC45 downregulation in GBM cells.
\end{abstract}

Conclusion: CircCDC45 promoted the progression of GBM by mediating the miR-485-5p/CSF-1 axis, and circCDC45 might be a promising plasmatic biomarker for GBM diagnosis and treatment.

Keywords: circCDC45, miR-485-5p, CSF-1, Glioblastoma

\section{Background}

Glioblastoma (GBM) is one of the common primary brain tumors [1]. The median survival time of GBM patients is only about 14.6 months, and almost all patients have a poor prognosis [2]. GBM, grade IV astrocytoma, is derived from multiple cell types with neural stem celllike properties, and it is almost unfulfillable to completely remove the entire GBM by surgery $[3,4]$. Partial

\footnotetext{
* Correspondence: wvuwgp@163.com

Department of Neurosurgery, Quzhou People's Hospital, No. 2, Zhongloudi, Kecheng District, Quzhou 324000, Zhejiang, China
}

GBM cells infiltrating into nearby brain tissues can easily cause tumor recurrence $[3,5]$. So GBM is considered to be one of the most aggressive and most difficult cancers to treat [6]. The identification and verification of prognosis and predictive biomarkers are of great significance for the understanding of tumor molecular characteristics and targeted therapy.

Circular RNAs (circRNAs) are characterized by a covalently closed loop with no free $3^{\prime}$ or $5^{\prime}$ end and belong to non-coding RNAs [7]. Emerging research reports that numerous circRNAs are deregulated in human cancers

(c) The Author(s). 2021 Open Access This article is licensed under a Creative Commons Attribution 4.0 International License, which permits use, sharing, adaptation, distribution and reproduction in any medium or format, as long as you give appropriate credit to the original author(s) and the source, provide a link to the Creative Commons licence, and indicate if changes were made. The images or other third party material in this article are included in the article's Creative Commons licence, unless indicated otherwise in a credit line to the material. If material is not included in the article's Creative Commons licence and your intended use is not permitted by statutory regulation or exceeds the permitted use, you will need to obtain permission directly from the copyright holder. To view a copy of this licence, visit http://creativecommons.org/licenses/by/4.0/ The Creative Commons Public Domain Dedication waiver (http://creativecommons.org/publicdomain/zero/1.0/) applies to the data made available in this article, unless otherwise stated in a credit line to the data. 
and closely modulate the progression of cancers [8]. CircRNAs can functionally either act as oncogenes or tumor suppressors in cancers with abundant and stable expression $[7,8]$ and thus are defined as effective biomarkers for cancer diagnosis and therapy [9]. CircRNA microarray identified a series of differently expressed circRNAs in GBM tissues compared to normal tissues [10, 11], which provided candidates for the following analyses. Although the vital role of circRNAs has been recognized, research on the function of circRNAs in cancer is still limited. Here, circCDC45, which was identified to be highly expressed in GBM tissues [11], caught our attention. CircCDC45, also named circ_0062270, was derived from $\mathrm{CDC} 45$ mRNA. The role and functional mechanism of circCDC45 in GBM are unknown and need to be further explored.

Competitive endogenous RNAs (ceRNAs) compete for microRNA (miRNA) response elements (MREs) to mediate the expression of miRNAs and downstream miRNA target genes [12]. Numerous studies demonstrated that circRNAs could serve as ceRNAs to mediate gene expression, which developed to be a canonical mechanism of circRNAs function in human cancers [13, 14]. MiR-485-5p was reported to play an anti-tumor role in glioma to block glioma cell proliferation and invasion [15]. Previous studies also mentioned that circAMOTL1 competed for miR-485-5p, regulating the development of cervical cancer [16]. However, the interaction between circCDC45 and miR-485-5p is not presented and is worth exploring.

MiRNAs generally combine with the 3' untranslated regions (3'UTR) of target mRNAs, triggering mRNA degradation and translation inhibition [17]. The 3'UTR of colony-stimulating factor 1 (CSF-1) harbors multiple unique motifs, and CSF-1 acting as a target of various miRNAs has been documented in cancers $[18,19]$. In addition, CSF-1 was extensively reported in glioma, involving in ionizing radiation resistance, high-grade of glioma, cell proliferation and invasion [20, 21]. Novel action mechanism of CSF-1 linked to miR-485-5p helps to enrich the role of CSF-1 in GBM.

Here, we investigated the function of circCDC45 in GBM both in vitro and in vivo. Besides, we predicted and validated the interaction between miR-485-5p and circCDC45 or CSF-1, thus providing a circCDC45/miR$485-5 \mathrm{p} / \mathrm{CSF}-1$ regulatory network to illustrate the function mechanism of circCDC45 in GBM. We aimed to provide promising biomarkers and strategies for GBM diagnosis and treatment.

\section{Methods}

\section{Specimen collection}

GBM specimens $(n=40)$ were collected from GBM patients, and normal brain tissues (NBTs) $(n=40)$ were collected from patients who underwent brain tissue resection of craniocerebral injury. All subjects were recruited from Quzhou People's Hospital. Besides, equal amount of plasma specimens was also collected from each subject, and plasma specimens from GBM patients before and after surgical resection (Pre-operation and Post-operation) were collected separately. Informed consent for specimen use was obtained from each participant. All specimens were promptly treated using liquid nitrogen after excising and then preserved at $-80^{\circ} \mathrm{C}$ conditions. This study was carried out with the approval of the Ethics Committee of Quzhou People's Hospital.

\section{Cell lines}

GBM cell lines, including U251, LN229, SHG-44, A172 and T98, and normal human astrocytes (NHA) were purchased from Bena Culture Collection (Beijing, China) and Procell Co., Ltd. (Wuhan, China). According to the culture method, all cells were cultured in 90\% Dulbecco's Modified Eagle Medium (DMEM; Gibco, Grand Island, NY, USA) plus $10 \%$ fetal bovine serum (FBS; Gibco) in an incubator at $37{ }^{\circ} \mathrm{C}$ containing $5 \% \mathrm{CO}_{2}$.

\section{Quantitative real-time polymerase chain reaction (qRT- PCR)}

Total RNA from specimens and cells was extracted using the Total RNA Kit (TianGen, Beijing, China). The synthesis of cDNA was performed using the cDNA firststrand synthesis kit (TianGen). For miRNA cDNA synthesis, reverse transcription was carried out using the miRcute miRNA cDNA first-strand synthesis kit (TianGen). Subsequently, qRT-PCR was implemented using the Talent qPCR SYBR Green Mix (TianGen). All experimental procedures were conducted following the protocols. The conditions used for $\mathrm{qRT}$-PCR were as it follows: $95^{\circ} \mathrm{C}$ for $10 \mathrm{~min}$ ( 1 cycle), $95^{\circ} \mathrm{C}$ for $10 \mathrm{~s}$ and $60^{\circ} \mathrm{C}$ for $1 \mathrm{~min}$ ( 40 cycles). Relative expression was computed using the $2^{-\Delta \Delta \mathrm{CT}}$ means with GAPDH or U6 as a house-keeping gene. The primer sequences were listed as follows: circCDC45, F: 5' -TTTGCACCAACCTCGT CATC-3' and R: 5' -GTCCTTCATCCGAACACACA-3'; CDC45, F: 5'-GAGTGGCTCTGGGAGTGAAC-3' and R: 5'-GGCTGACGATGTCCCATGAT-3'; miR-485-5p, F: 5'-GGAGAGGCTGGCCGTGAT-3' and R: 5'-CAGT GCGTGTCGTGGAGT-3'; CSF-1, F: 5'-ACCCCTCC ACCCTCTCTG-3' and R: $5^{\prime}$-CTGCCCCTTCACTTGC TG-3'; GAPDH, F: 5'-AAGTATGACAACAGCCTCAA GA-3' and R: 5' -CACCACCTTCTTGATGTCATCA-3'; U6, F: 5'-CTCGCTTCGGCAGCACA-3' and R: 5'AACGCTTCACGAATTTGCGT-3'.

\section{Actinomycin D treatment}

GBM cells were treated with Actinomycin D $(50 \mathrm{ng} / \mathrm{mL}$; Cell Signaling Technology, Danvers, MA, USA) and 
maintained for 8,16 and $24 \mathrm{~h}$ at room temperature. Cells at different time points were harvested and used for qRT-PCR to monitor the expression of circCDC45 and CDC45 mRNA. Three independent experiments were conducted.

\section{RNase $\mathrm{R}$ digestion}

The isolated RNAs from GBM cells were exposed to

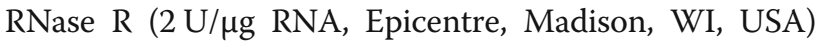
for $15 \mathrm{~min}$ at $37^{\circ} \mathrm{C}$. Then, total RNA was used for qRTPCR analysis to detect the expression of circCDC45 and CDC45 mRNA. Untreated RNA (Mock) was used as control. Three independent experiments were conducted.

\section{Cell transfection}

Small interference RNA (siRNA) was used for expression silencing. In brief, siRNA targeting circCDC45 (sicircCDC45\#1 and si-circCDC45\#2), siRNA targeting CSF-1 (si-CSF-1\#1 and si-CSF-1\#2) and siRNA negative control (si-NC) were constructed by GenePharma (Shanghai, China). pCDNA3.1(+) CircRNA Mini Vector was used for circCDC45 overexpression (pcDNAcircCDC45), and fusion plasmid was constructed by Ke Lei BioTech Co., Ltd. (Shanghai, China) with empty pcDNA vector as a negative control (pcDNA-NC). MiR485-5p mimics (miR-485-5p), miR-485-5p inhibitors (anti-miR-485-5p) and their separate control (miR-NC and anti-miR-NC) were all obtained from Ribobio (Guangzhou, China). The oligonucleotides (siRNA: 200 $\mathrm{nM}$; mimic and inhibitor: $100 \mathrm{nM}$ ) or fusion plasmids $(1 \mu \mathrm{g})$ were transfected into cells using Lipofectamine 3000 (Invitrogen, Carlsbad, CA, USA) in line with detailed usage instructions.

\section{Cell counting kit-8 (CCK-8) assay}

GBM cells were plated in 96-well plates (2000 cells/well) with different transfection. After culturing for 24, 48 and $72 \mathrm{~h}$, cells were exposed to $10 \mu \mathrm{L}$ CCK- 8 reagent (Beyotime, Shanghai, China) and cultured for continuing $4 \mathrm{~h}$. The absorbance at $450 \mathrm{~nm}$ of GBM cells at different time points was examined using a microplate reader (BioRad, Hercules, CA, USA). Each sample was in triplicate, and three independent experiments were conducted.

\section{Colony formation assay}

GBM cells were cultured in 6-well plates $(10 \mathrm{~cm}$ diameter) with different transfection, at a density of 200 cells/ plate. The plates were cultured at $37{ }^{\circ} \mathrm{C}$ conditions containing 5\% $\mathrm{CO}_{2}$. After 2 weeks, formed colonies were fixed using methanol and then stained using $0.1 \%$ crystal violet followed by photograph under a microscope (Olympus, Tokyo, Japan). Each sample was in triplicate, and three independent experiments were conducted.

\section{Western blot}

Total proteins were extracted using RIPA Lysis Buffer (Beyotime) and next separated by $10 \%$ sodium dodecyl sulfate-polyacrylamide gel electrophoresis (SDS-PAGE). The separated proteins were transferred onto polyvinylidene difluoride (PVDF) membranes (Bio-Rad) and incubated with the blocking buffer. Subsequently, the membranes containing protein bands were probed with the primary antibodies against Ki67 (ab231172; Abcam, Cambridge, MA, USA), Proliferating cell nuclear antigen (PCNA; ab18197; Abcam), CSF-1 (ab99178; Abcam) and GAPDH (ab9485; Abcam) at $4{ }^{\circ} \mathrm{C}$ overnight and subsequently treated with the secondary antibodies (ab205718; Abcam). The protein bands were imaged using an enhanced chemiluminescence kit (ECL; Beyotime). Each sample was in triplicate, and three independent experiments were conducted.

\section{Transwell assay}

Cell migration and cell invasion capacities were monitored using transwell chambers (BD Biosciences, San Jose, CA, USA). For invasion analysis, GBM cells $(5 \times$ $10^{4}$ cells) in $150 \mu \mathrm{L}$ serum-free medium were planted into the top of chambers pre-coated with Matrigel (BD Biosciences). For migration analysis, GBM cells $\left(1 \times 10^{4}\right.$ cells) in serum-free medium were added into the upper chambers with nothing. Meantime, $600 \mu \mathrm{L}$ fresh DMEM containing 10\% FBS was filled with the lower chambers. After 24-h incubation, cells remaining on the upper membrane were removed with a cotton swab, and the lower cells were fixed in methanol and then stained using $0.1 \%$ crystal violet followed by observation and photograph using a microscope (Olympus) with 5 randomly selected fields (magnification 100x). Each sample was in triplicate, and three independent experiments were conducted.

\section{Bioinformatics analysis}

We used the bioinformatics databases, including starBase (http://starbase.sysu.edu.cn/) and circbank (http:// www.circbank.cn/), to predict the potential miRNAs targeted by circCDC45, and starBase was utilized to predict the potential mRNAs targeted by miR-485-5p.

\section{Dual-luciferase reporter assay}

Luciferase reporter plasmids were assembled in pmirGLO vector (Promega, Madison, WI, USA) containing the wild-type sequence of circCDC45 (harboring miR485-5p binding sites) or containing the mutant-type sequence of circCDC45 (harboring mutated miR-485-5p binding sites). The fusion plasmids were named as circCDC45-WT and circCDC45-MUT, respectively. Similarly, the fusion plasmids, CSF-1 3'UTR-WT and CSF-1 3'UTR-MUT were also generated in the same 
method. LN229 and A172 cells were then cotransfected with miR-485-5p $(50 \mathrm{nM})$ or miR-NC $(50 \mathrm{nM})$ together with circCDC45-WT, circCDC45-MUT, CSF-1 3'UTRWT and CSF-1 3'UTR-MUT (200 $\mu$ g) using Lipofectamine 3000 and cultured for $48 \mathrm{~h}$. Luciferase activity was ascertained in LN229 and A172 cells using the DualLuciferase Assay System (Promega). Three independent experiments were conducted.

\section{RNA immunoprecipitation (RIP) assay}

LN229 and A172 cells were lysed using the lysis buffer from the Magna RIP Kit (Millipore), and cell lysates were then incubated with RIP buffer containing magnetic beads conjugated with Argonaute 2 antibody (antiAgo2) or Immunoglobulin G antibody (anti-IgG; control) at $4{ }^{\circ} \mathrm{C}$ overnight. Afterwards, RNA was eluted and purified, and immunoprecipitated RNA was analyzed by qRT-PCR. Three independent experiments were conducted.

\section{In vivo experiments}

A total of $12 \mathrm{BALB} / \mathrm{C}$ nude mice (Male, 4-6-week-old, 16-20 g) were purchased from Beijing HFK Bioscience Co., Ltd. (Beijing, China) and randomly divided into 2 groups ( $n=6$ per group). Short hairpin RNA (shRNA) lentiviral vector containing circCDC45 (sh-circCDC45), and its negative controls (sh-NC) were synthesized by GeneCopoeia (Guangzhou, China). LN229 cells (100 $\mu \mathrm{L}$; $1 \times 10^{6}$ cells) transfected with sh-circCDC45 or sh-NC were subcutaneously implanted into nude mice. Mice were adaptively raised for 1 week to allow tumor growth. Then, tumor volume was recorded once a week, lasting 6 weeks. After 6 weeks, all mice were sacrificed to collect tumor tissues for the following analysis. All animal experimental procedures were approved by the Animal Care and Use Committee of Quzhou People's Hospital.

\section{Statistical analysis}

All experiments were conducted at least three times. Differences between two groups were analyzed by Student's $t$-test, and differences among $\geq$ three groups were analyzed by analyses of variance with Tukey post hoc test. The expression correlation in clinical tissues between two variables was performed using Spearman's correlation test. Statistical analyses were carried out using GraphPad Prism 7 (GraphPad Software, Inc., La Jolla, CA, USA). $P$ value $\leq 0.05$ was deemed to be statistically significant. The data were shown as the mean \pm standard deviation.

\section{Result}

\section{CircCDC45 was aberrantly highly expressed in GBM} tissues and cells

The microarray data of circRNAs that were differently expressed in GBM tissues and non-tumor tissues were deposited in the Gene Expression Omnibus (GEO) database (accession: GSE109569; https://www.ncbi.nlm.nih. gov/geo/query/acc.cgi?acc=GSE109569), and we downloaded the data and analyzed the top 5 circRNAs significantly downregulated or upregulated in GBM tissues. Heat map showed that circCDC45 (has_circ_0062270) is one of the significantly upregulated circRNAs in GBM tissues (Fig. 1A). CircCDC45 was derived from linear CDC45 mRNA, and the schematic diagram was depicted to describe the formation of circCDC45 (Fig. 1B). The size of circCDC45 in tumor tissues and normal tissues was determined by PCR, and a band with about $385 \mathrm{bp}$ was presented in gel (Fig. 1C). Then we verified the expression of circCDC45 in clinical specimens by qRT$\mathrm{PCR}$, and the data displayed that the expression of circCDC45 was noticeably promoted in GBM tissues $(n=40)$ compared with that in NBT tissues $(n=40)$ (Fig. $1 C)$. Likewise, the expression of circCDC45 was also elevated in GBM cell lines (U251, LN229, SHG-44 and T98) compared with that in normal glial cells (NHA) (Fig. 1D). LN229 and A172 cells were chosen for the following in vitro analyses because the expression of circCDC45 in these two cell lines was relatively higher than other GBM cell lines. Besides, Actinomycin D treatment notably weakened the expression of CDC45 mRNA expression but had negligible effects on circCDC45 expression (Fig. 1E and F). RNase R treatment also notably impaired the expression of CDC45 mRNA expression but hardly weakened the expression of circCDC45 (Fig. 1G and H). These data hinted that circCDC45 was dysregulated in GBM and might take part in GBM progression.

\section{CircCDC45 downregulation blocked GBM cell proliferation, migration and invasion}

Loss-function of circCDC45 was conducted to explore the role of circCDC45 in GBM cells. After the transfection of si-circCDC45\#1 or si-circCDC45\#2, the expression of circCDC45 was significantly declined in LN229 and A172 cells (Fig. 2A and B). CircCDC45 downregulation markedly repressed GBM cell proliferation by the analyses of CCK-8 and colony formation (Fig. 2C, D and E). Besides, cell proliferation-related proteins, including Ki67 and PCNA, were quantified, and the data showed that both Ki67 and PCNA were significantly downregulated in LN229 and A172 cells transfected with sicircCDC45 (Fig. 2F). The raw images of western blot data for the bands of Ki67 and PCNA were shown in Additional file 1: Supplementary file. CircCDC45 downregulation also suppressed GBM cell invasion and migration by transwell assay (Fig. 2G and $\mathrm{H}$ ). These data suggested that circCDC45 downregulation inhibited GBM malignant behaviors in vitro. 

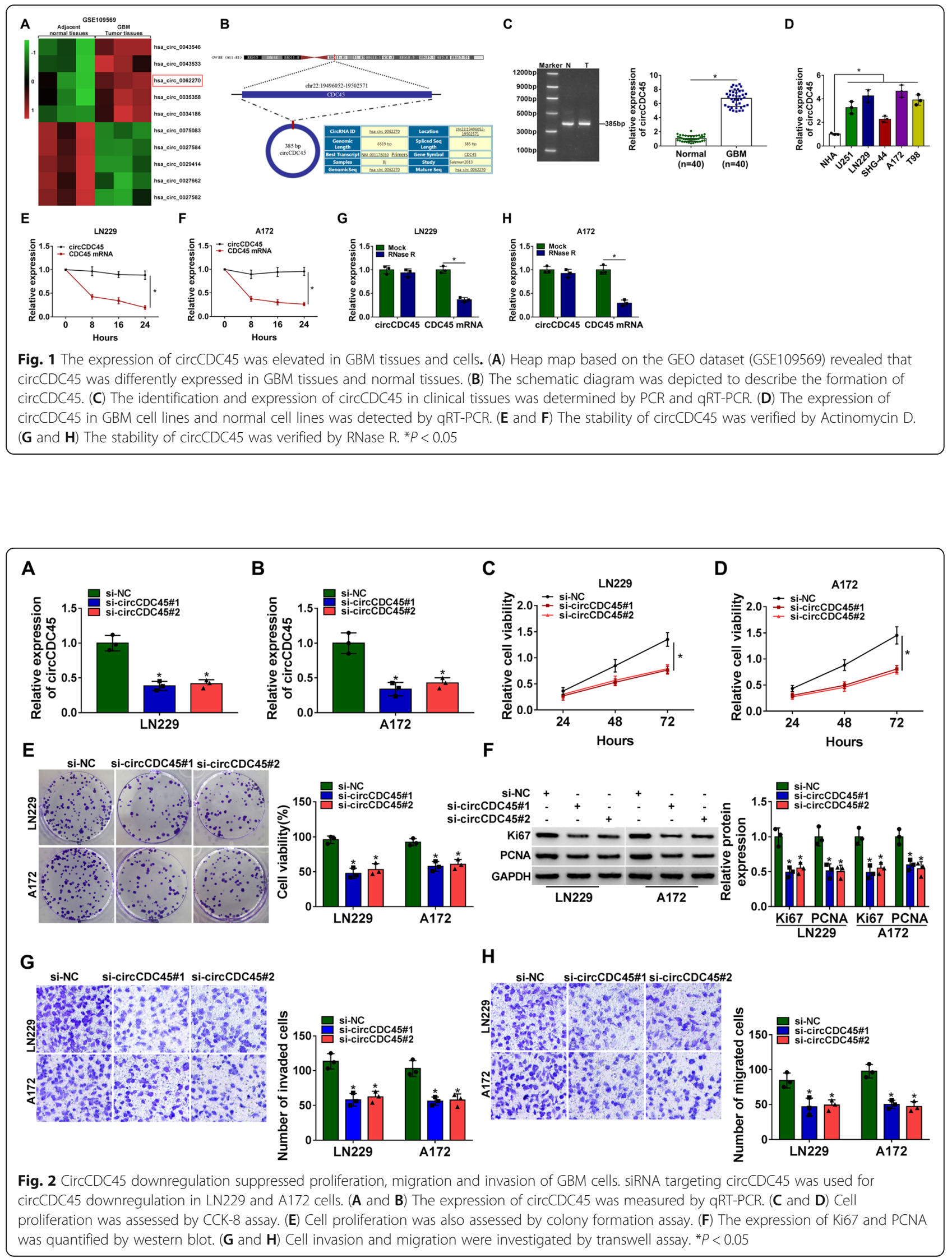
CircCDC45 functioned as the molecular sponge of miR485-5p

The view that certain circRNAs can function as decoys or sponges to suppress miRNA expression and degrade miRNA function is canonical [22]. To identify that circCDC45 harbored the same property, we predicted the potential target miRNAs using starBase and circbank. A total of 18 miRNAs targeted by circCDC45 were obtained from starBase, and a total of 35 miRNAs targeted by circCDC45 were obtained from circbank, including 6 miRNAs (miR-218-5p, miR-125a-5p, miR-5255p, miR-485-5p, miR-6088 and miR-2278) in both starBase and circbank (Fig. 3A). We focused on these 6 miRNAs and examined their expression in LN229 and A172 cells with circCDC45 overexpression. Interestingly, the expression of miR-485-5p decreased most (Fig. 3B and C). We deduced that miR-485-5p was likely a target of circCDC45. To further validate the interaction between miR-485-5p and circCDC45, the mutant sequence of circCDC45 with miR-485-5p binding sites was generated according to its wild sequence (Fig. 3D). Dualluciferase reporter assay showed that a decrease of luciferase activity was observed in LN229 and A172 cells with cotransfection of miR-485-5p and circCDC45-WT (Fig. 3E and F). In addition, RIP assay presented that both circCDC45 and miR-485-5p were significantly enriched in the Anti-Ago2 group compared with that in the Anti-IgG group (Fig. 3G and H). In LN229 and A172 cells with circCDC45 knockdown, the expression of miR-485-5p was remarkably enhanced (Fig. 3I). Moreover, the expression of miR-485-5p was remarkably declined in GBM clinical specimens and GBM cell lines compared with that in NBT specimens and NHA cells, respectively (Fig. 3J and K), and miR-485-5p expression was negatively correlated with circCDC45 expression in GBM clinical tissues (Fig. 3L). All data manifested that miR-485-5p was a target of circCDC45, and the expression pattern of miR-485-5p in GBM was opposite to circCDC45.

\section{CircCDC45 regulated GBM cell proliferation, migration and invasion by targeting miR-485-5p}

LN229 and A172 cells with circCDC45 knockdown alone or circCDC45 and miR-485-5p knockdown together were used to test the contrary role of circCDC45 and miR-485-5p. The expression of miR-485-5p was

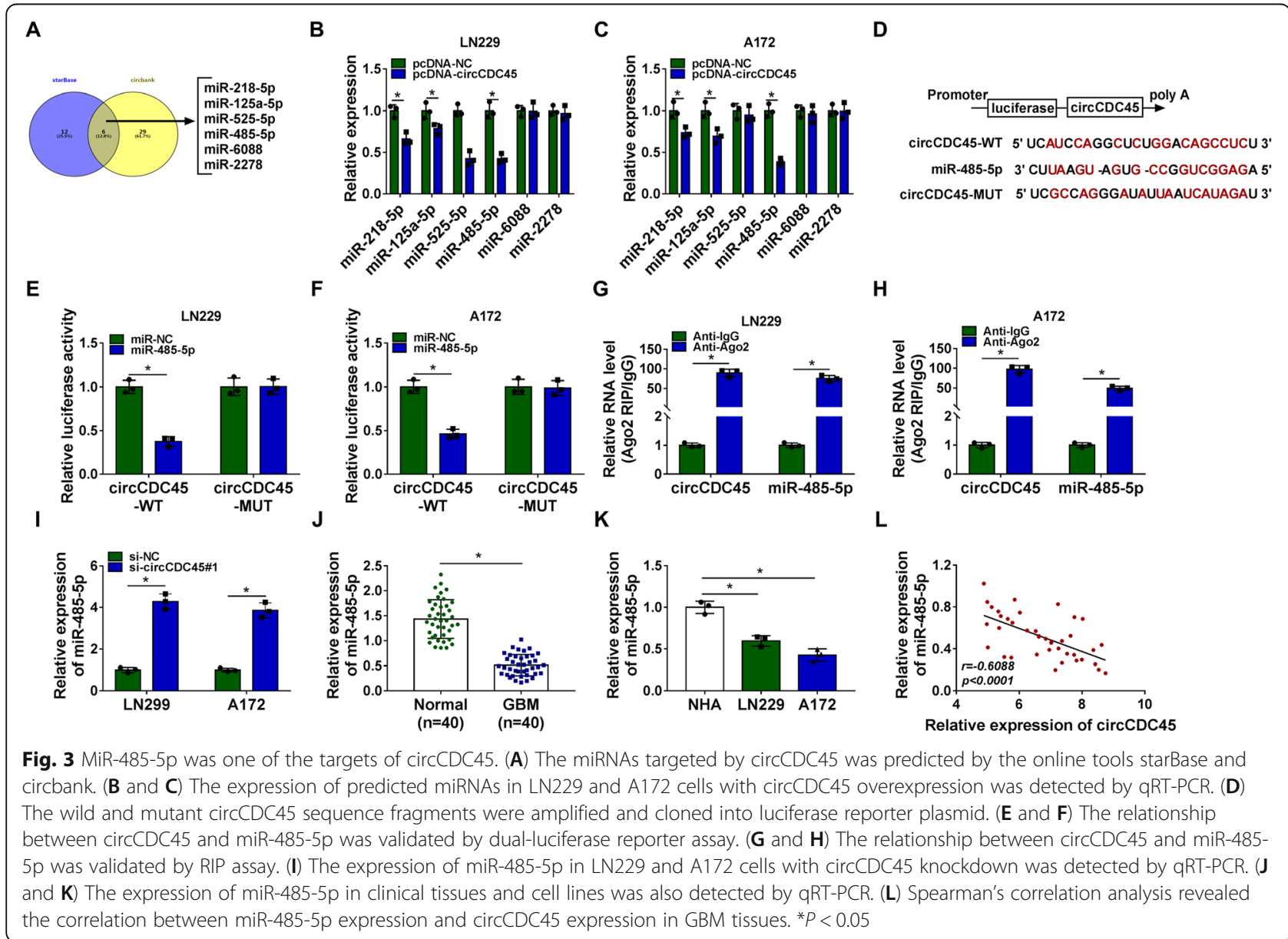


significantly promoted in LN229 and A172 cells transfected with si-circCDC45 but significantly lessened in cells transfected with si-circCDC45 + anti-miR-485-5p (Fig. 4A). In function, OD value and colony number were blocked by circCDC45 downregulation, while the inhibition of miR-485-5p substantially recovered OD value and colony number (Fig. $4 \mathrm{~B}$ and $\mathrm{C}$ ). The expression of Ki67 and PCNA impaired in LN229 and A172 cells transfected with si-circCDC45 was largely restored in cells transfected with si-circCDC45 + anti-miR-485-5p (Fig. 4D and E). The number of invaded and migrated cells was weakened by circCDC45 downregulation alone but promoted by circCDC45 downregulation combined with miR-485-5p inhibition in LN229 and A172 cells (Fig. 4F and G). The data suggested that miR-485-5p inhibition could promote GBM cell malignant behaviors blocked by circCDC45 downregulation.

\section{CSF-1 was a target of miR-485-5p}

MiRNAs generally bound to the 3' untranslated region (3'UTR) of mRNAs to post-transcriptionally regulated miRNA expression. Therefore, we predicted the potential mRNAs targeted by miR-485-5p, and the tool starBase exhibited that there were binding sites between miR-485-5p and CSF-1 3'UTR (Fig. 5A). The mutant sequence of CSF-1 3'UTR and the wild sequence of CSF-1 3'UTR were amplified and cloned into luciferase reporter plasmid, and we found miR-485-5p restoration strikingly reduced the luciferase activity of LN229 and A172 cells transfected with CSF-1 3'UTR-WT (Fig. 5B and C). RIP
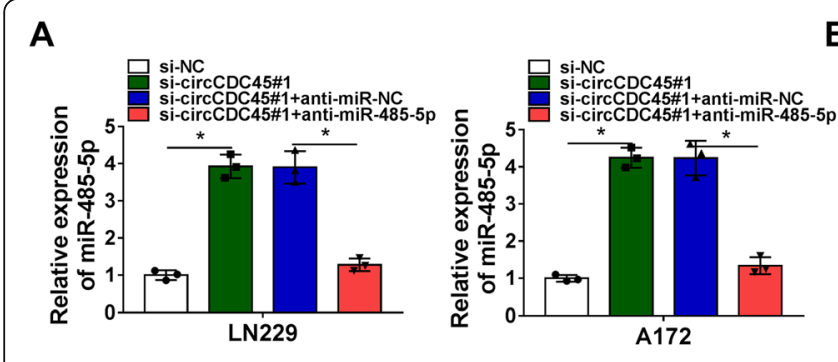

B

C
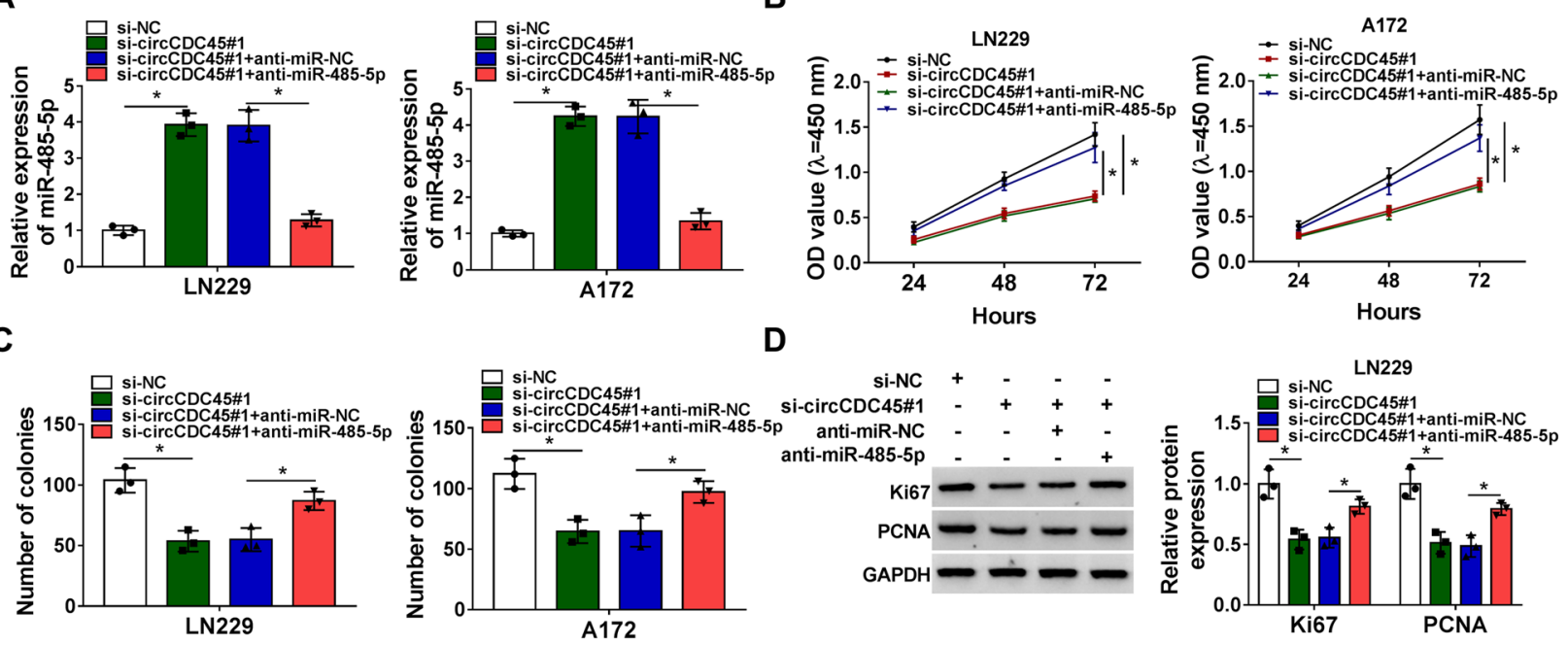

E
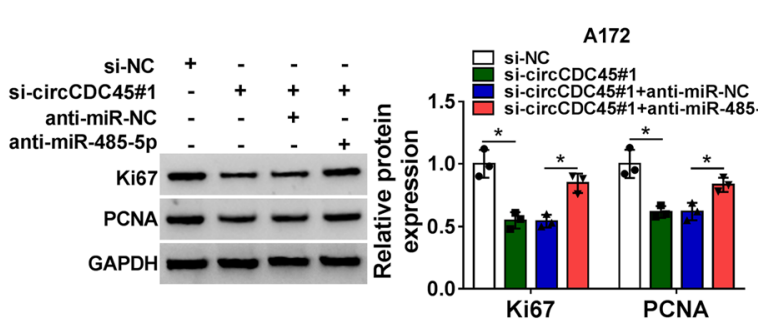

$\mathbf{F}$
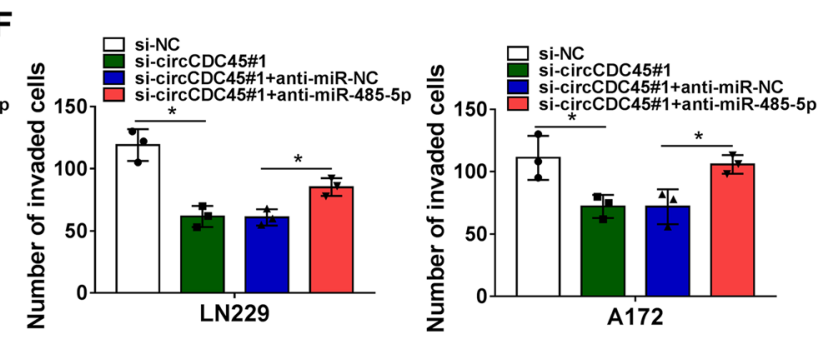

G

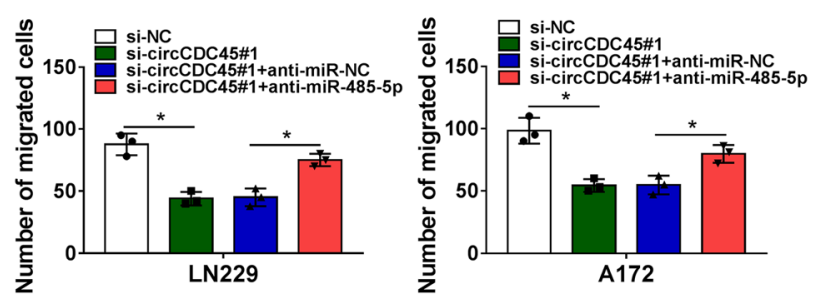

Fig. 4 MiR-485-5p inhibition reversed the effects of circCDC45 downregulation. LN229 and A172 cells were transfected with si-circCDC45 or sicircCDC45 + anti-miR-485-5p with si-NC or si-circCDC45 + anti-miR-NC as the separate control. (A) The expression of miR-485-5p in these transfected cells was examined using qRT-PCR. (B and C) Cell proliferation was assessed by CCK-8 assay and colony formation assay. (D and E) The expression of Ki67 and PCNA was quantified by western blot. (F and $\mathbf{G})$ Cell invasion and cell migration were investigated by transwell assay. ${ }^{*} P<0.05$ 


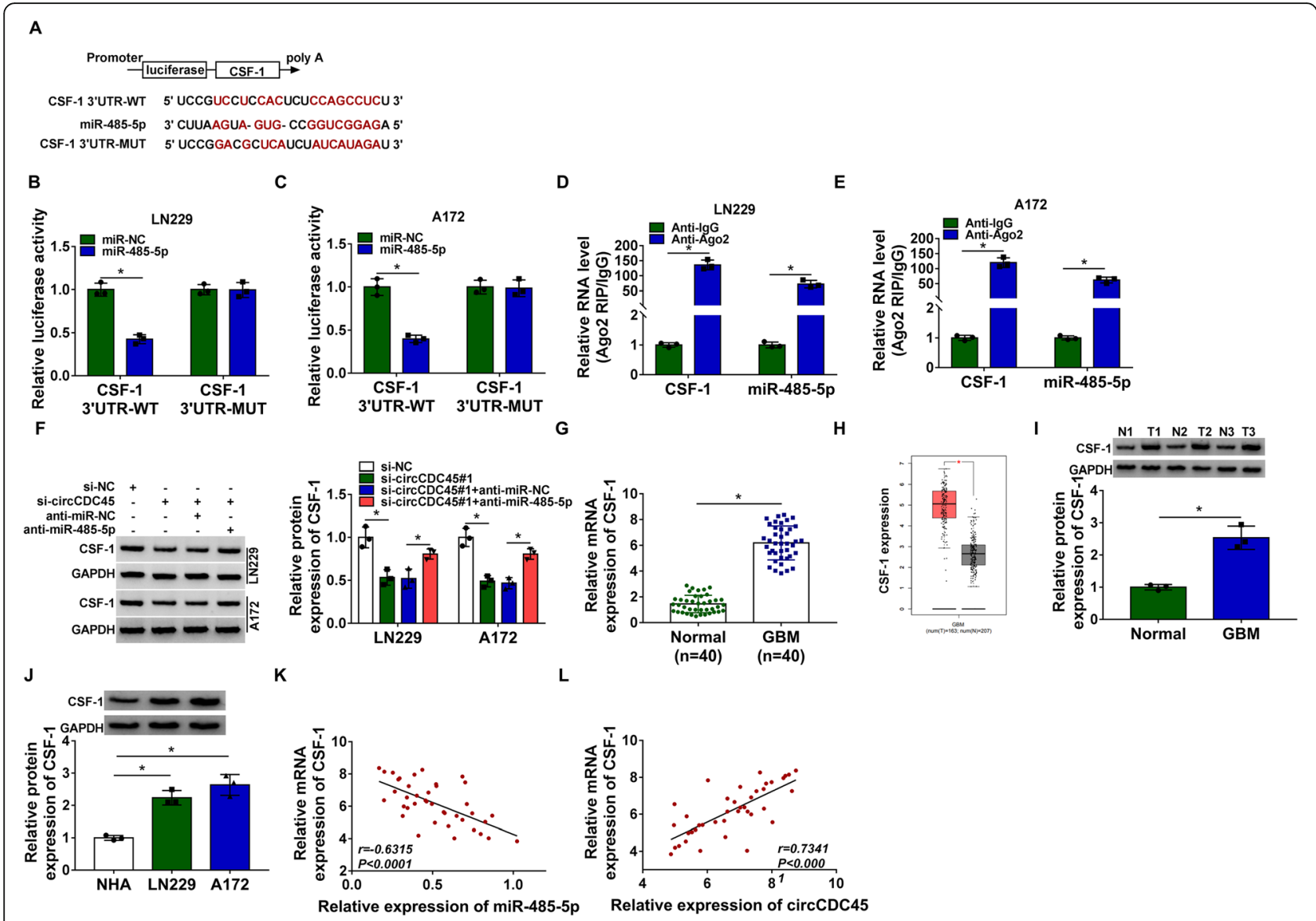

Fig. 5 MiR-485-5p directly bound to CSF-1. (A) CSF-1 was predicted as a target of miR-485-5p by starBase, and the wild and mutant sequences of CSF-1 3'UTR were designed for dual-luciferase reporter assay. (B and C) The interaction between LN229 and A172 was validated by dual-luciferase reporter assay. (D and $\mathbf{E}$ ) The interaction between LN229 and A172 was validated by RIP assay. (F) The expression of CSF-1 in LN229 and A172 cells transfected with si-circCDC45, si-NC, si-circCDC45 + anti-miR-485-5p or si-circCDC45 + anti-miR-NC was checked by western blot. (G) The expression of circCDC45 in clinical tissues was checked using qRT-PCR. (H) The expression of circCDC45 in GBM tissues was obtained from the TCGA database. (I) The expression of circCDC45 in clinical specimens was checked by western blot. (J) The expression of circCDC45 in NHA, LN229 and A172 cells was examined using western blot. (K and L) Spearman's correlation analysis revealed the correlation between CSF-1 expression and miR-485-5p expression or circCDC45 expression in GBM tissues. ${ }^{*} P<0.05$

assay presented that both miR-485-5p and CSF-1 were enriched in the Anti-Ago2 group compared with that in the Anti-IgG group (Fig. 5D and E). In addition, we found that the expression of CSF-1 was decreased in LN229 and A172 cells transfected with si-circCDC45 but promoted in cells transfected with si-circCDC45 + anti-miR-485-5p (Fig. 5F), suggesting that circCDC45 could regulate the expression of CSF-1 by sponging miR-485-5p. Moreover, the expression of CSF-1 was significantly enhanced in GBM tissues $(n=40)$ compared with that in the NBTs $(n=40)$, which was consistent with the data from TCGA database (Fig. 5G, H and I). Likewise, the expression of CSF-1 was elevated in LN229 and A172 cells compared with that in NHA cells (Fig. 5J). Correlation analysis revealed that CSF-1 expression was negatively correlated with miR-485-5p expression but positively correlated with circCDC45 expression in
GBM tissues (Fig. 5K and L). The data suggested that CSF-1 was a target of miR-485-5p, and circCDC45 regulated CSF-1 by targeting miR-485-5p.

\section{CSF-1 knockdown blocked GBM cell proliferation, migration and invasion}

The expression of CSF-1 was notably decreased in LN229 and A172 cells transfected with si-CSF-1\#1 or siCSF-1\#2 (Fig. 6A and B). Then, LN229 and A172 cells with CSF-1 knockdown were used for functional analysis. CCK-8 assay and colony formation assay indicated that CSF-1 knockdown strikingly restrained cell proliferation (Fig. 6C, D and E). The expression of Ki-67 and PCNA was notably decreased in cells with CSF-1 knockdown (Fig. 6F). Moreover, transwell assay displayed that the ability of cell migration and invasion were restricted 


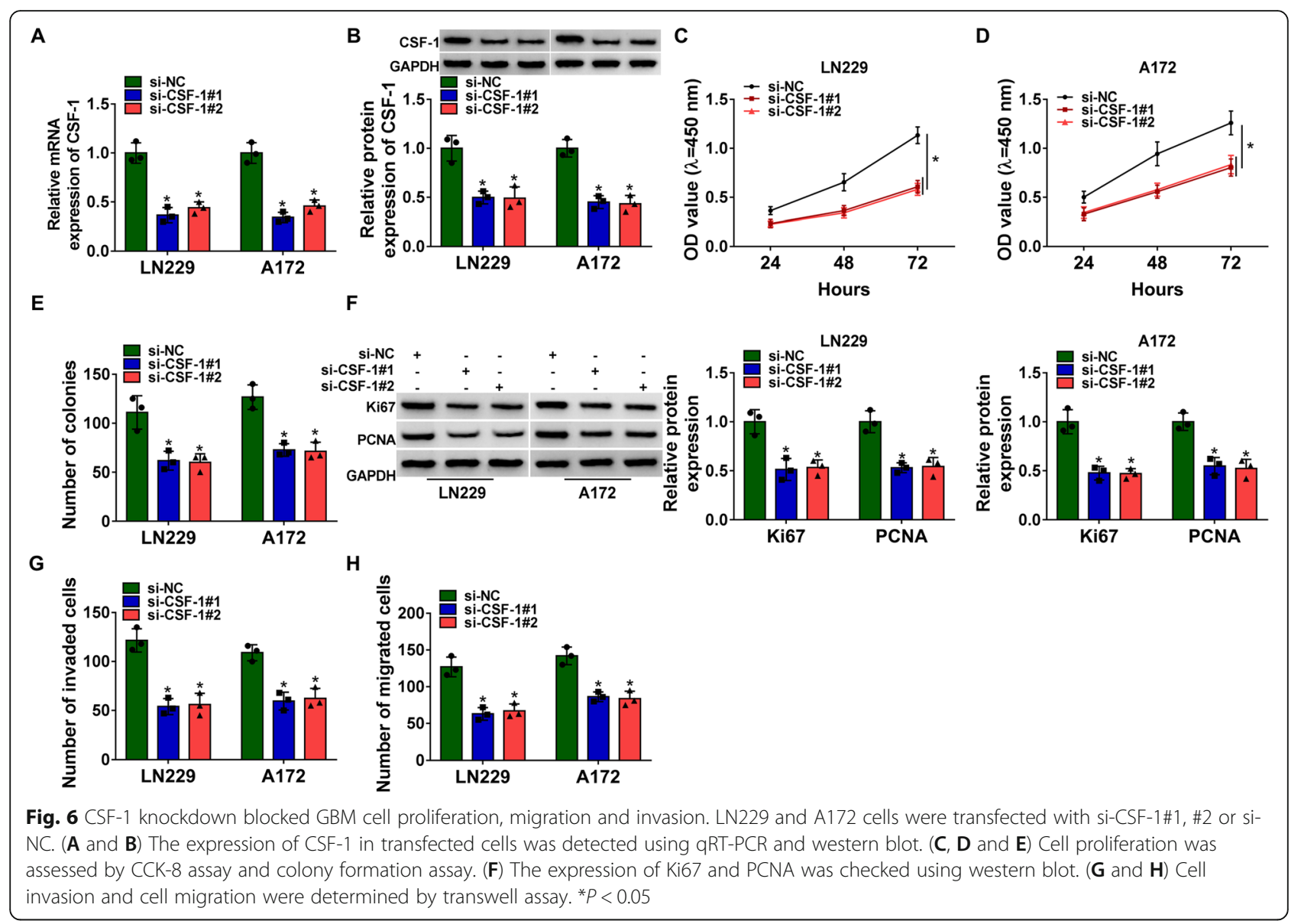

A

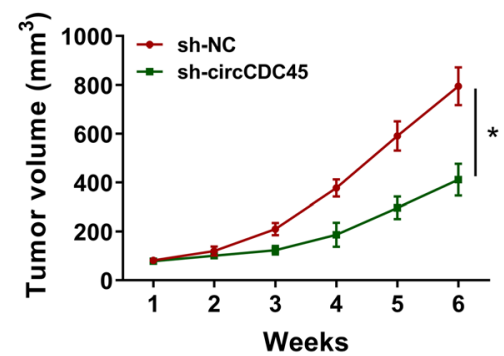

D

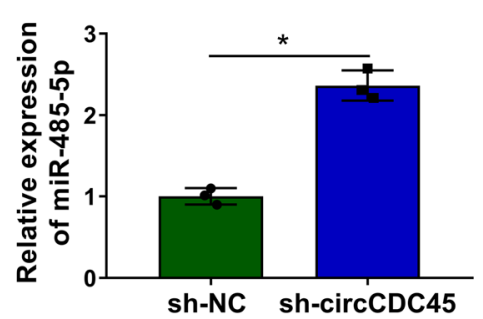

B

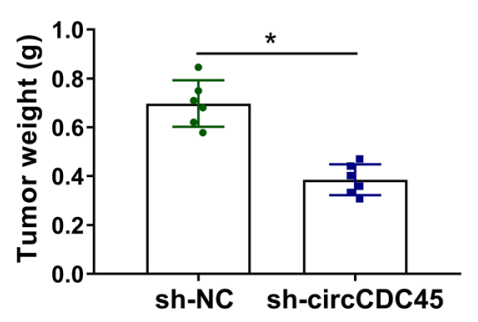

E

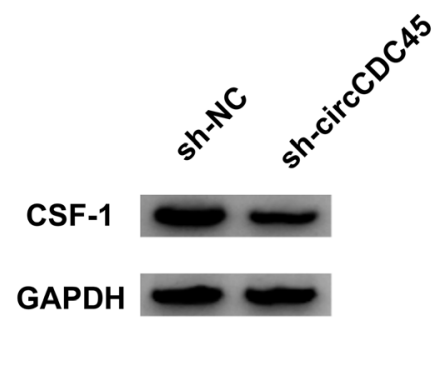

C
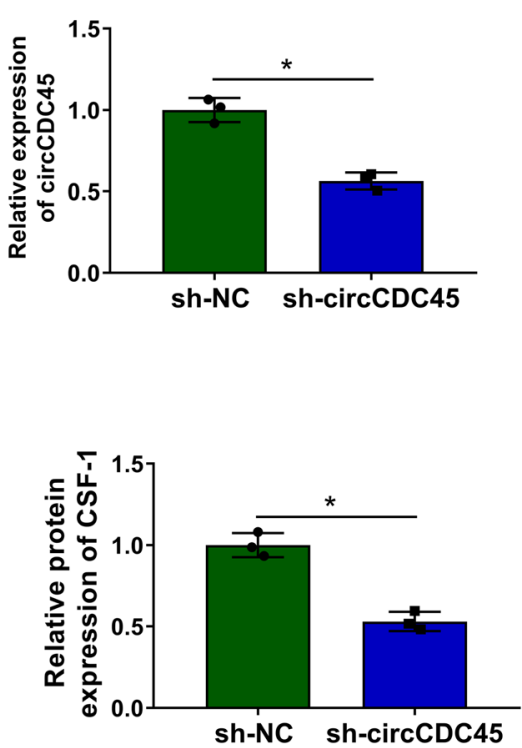

Fig. 7 CircCDC45 regulated tumor growth in vivo by targeting the miR-485-5p/CSF-1 axis. (A and B) The effects of circCDC45 knockdown on tumor volume and tumor weight. (C and D) The expression of circCDC45 and miR-485-5p in the excised tissues was checked using qRT-PCR. (E) The expression of CSF-1 in the excised tissues was checked using western blot. ${ }^{*} P<0.05$ 
by CSF-1 knockdown (Fig. 6G and H). All data suggested that CSF-1 might be an oncogene in GBM.

\section{CircCDC45 downregulation repressed tumor growth in vivo by targeting the miR-485-5p/CSF-1 axis}

We further investigated the role of circCDC45 in vivo. LN229 cells transfected with sh-circCDC45 or sh-NC were used for the tumorigenic test. The result showed that circCDC45 deficiency dramatically weakened tumor growth, including tumor volume and tumor weight (Fig. 7A and B). In the excised tumor tissues, we examined the expression of circCDC45, miR-485-5p and CSF1. Compared to sh-NC, the expression of circCDC45 and CSF-1 was remarkably declined, while the expression of miR-485-5p was remarkably increased in the tissues from the sh-circCDC45 group (Fig. 7C, D and E). The data circCDC45 also played functions in vivo through the miR-485-5p/CSF-1 axis.

\section{Discussion}

GBM is characterized by high invasion and reoccurrence, and frequent recurrence and invasion of GBM make it resistant to chemotherapy and radiation therapy [4]. GBM remains conferring poor outcomes with limited therapeutic progress. Biomarker-based projects have aroused much interest in precision medicine and targeted therapy [23]. Emerging evidence has identified circRNAs as novel biomarkers, and the investigation of specific circRNAs involved in tumorigenesis of GBM is imperative.

Previous studies showed that circFOXO3 enrichment accelerated GBM cell invasion by functioning as a "ceRNA" to mediate the miR-138-5p\&miR-432-5p/ NFAT5 network [24]. Circ_0029426 overexpression was linked to unfavorable prognosis of GBM, and circ 0029426 restoration induced GBM cell proliferation but blocked cell apoptosis [25]. High expression of CircSMARCA5 was linked to high overall survival, and circSMARCA5 regulated angiogenesis in GBM multiforme by binding to SRSF1 [26]. In our research, we investigated the function of circCDC45 in GBM and defined it as an oncogene. The evidence was that circCDC45 downregulation impaired GBM cell proliferation, invasion and migration in vitro and tumor development in vivo. CircCDC45 was monitored to be markedly upregulated in GBM tissues by circRNA microarray [11]. Besides, a recent study insisted that high expression of circCDC45 was closely linked to large tumor size, high grade and poor survival in glioma and contributed to glioma cell growth and metastasis [27]. All findings manifested that the inhibition of circCDC45 might be a novel strategy against GBM progression.

For mechanism analysis, we obtained potential target miRNAs of circCDC45, and miR-485-5p was indeed targeted by circCDC45 through multiple validations. A former study identified that miR-485-5p served as an anti-tumor role in glioma, and miR-485-5p overexpression glioma tumorigenesis by repressing the expression of its target gene TPD52L2 [15]. Nayak et al. discovered that restoration of miR-485-5p impaired GBM cell invasion and induced apoptosis [28]. Consistent with these findings, we discovered that the abundance of miR-485$5 p$ was weak in GBM tissues and cells. MiR-485-5p inhibition counteracted the effects of circCDC45 downregulation to induce GBM cell proliferation, migration and invasion, while miR-485-5p restoration blocked these malignant behaviors. Similarly, miR-485-5p as a tumor suppressor was also recorded in other cancers, including hepatocellular carcinoma, cholangiocarcinoma and esophageal cancer $[4,29,30]$, indicating that miR-485$5 \mathrm{p}$ was a well-recognized tumor suppressor in various cancers.

Furthermore, we noticed that miR-485-5p bond to CSF-1 3'UTR, and further assay verified that CSF-1 was indeed targeted by miR-485-5p. The tumorigenicity of CSF-1 was widely documented in various cancers, and high expression of CSF-1 predicted poor prognosis [31, 32]. Likewise in GBM, De et al. held the view that CSF-1 was frequently overexpression in human cancers, and overexpression of CSF-1 facilitated the formation of high-grade glioma [20]. Besides, CSF-1 overexpression promoted glioma cell viability and metastasis, while CSF-1 knockdown

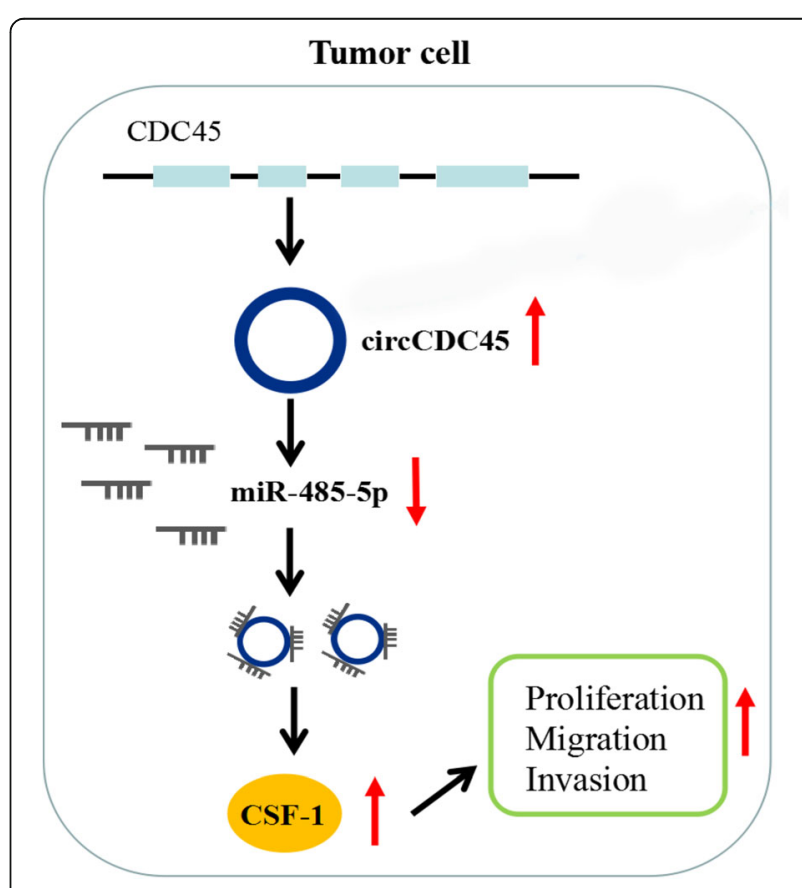

Fig. 8 Schematic diagram revealed that the circCDC45/miR-485-5p/ CSF-1 axis participated in the development of GBM 
presented the opposite effects [33]. Consistent with these consequences, we viewed that the expression of CSF-1 was abnormally strengthened in GBM tissues and cells, and CSF-1 knockdown repressed GBM cell proliferation, invasion and migration. Additionally, CSF-1 expression was negatively linked to miR-485-5p expression but positively linked to circCDC45 expression in GBM tissues, and circCDC45 downregulation weakened CSF-1 expression, while miR-485-5p inhibition recovered CSF-1 expression, suggesting that circCDC45 regulated CSF-1 by targeting miR-485-5p.

\section{Conclusions}

CircCDC45, derived from CDC45 mRNA, positively regulated the expression of CSF-1 by targeting miR-485-5p, thereby promoting GBM cell proliferation, migration and invasion and contributing to the progression of GBM (Fig. 8). Hence, circCDC45 was a promising biomarker for GBM diagnosis and treatment.

\section{Abbreviations}

GBM: Glioblastoma; circRNAs: Circular RNAs; CSF-1: Colony-stimulating factor 1; qRT-PCR: Quantitative real-time polymerase chain reaction; CCK-8: Cell counting kit - 8; RIP: RNA immunoprecipitation; circRNAs: Circular RNAs; ceRNAs: Competitive endogenous RNAs; miRNA: MicroRNA; MREs: MicroRNA response elements; 3'UTR: 3' untranslated regions

\section{Supplementary Information}

The online version contains supplementary material available at https://doi. org/10.1186/s12885-021-08803-7.

Additional file 1 Supplementary file. The raw data of western blot for the expression levels of Ki67 and PCNA.

\section{Acknowledgements}

None.

\section{Authors' contributions}

$R L$ conceived and designed the study. WD performed the experiments. AW and $R L$ analyzed the data. YL the manuscript. All authors read and approved the final manuscript.

\section{Funding}

None.

\section{Availability of data and materials}

All data generated or analysed during this study are included in this published article [and its supplementary information files].

\section{Declarations}

\section{Ethics approval and consent to participate}

This study was carried out with the approval of the Ethics Committee of Quzhou People's Hospital. Written informed consent was obtained from all individual participants included in the study.

\section{Consent for publication}

Not Applicable.

\section{Competing interests}

The authors declare that they have no financial conflicts of interest.
Received: 3 November 2020 Accepted: 22 September 2021

Published online: 09 October 2021

\section{References}

1. Ostrom QT, et al. CBTRUS statistical report: primary brain and central nervous system tumors diagnosed in the United States in 2007-2011. Neuro Oncol. 2014;16(Suppl 4):iv1-63.

2. Hottinger AF, Stupp R, Homicsko K. Standards of care and novel approaches in the management of glioblastoma multiforme. Chin J Cancer. 2014;33(1): 32-9. https://doi.org/10.5732/cjc.013.10207.

3. Taylor MA, Das BC, Ray SK. Targeting autophagy for combating chemoresistance and radioresistance in glioblastoma. Apoptosis. 2018; 23(11-12):563-75. https://doi.org/10.1007/s10495-018-1480-9.

4. Davis ME. Glioblastoma: overview of disease and treatment. Clin J Oncol Nurs. 2016:20(5 Suppl):S2-8. https://doi.org/10.1188/16.CJON.S1.2-8.

5. Lau D, Magill ST, Aghi MK. Molecularly targeted therapies for recurrent glioblastoma: current and future targets. Neurosurg Focus. 2014;37(6):E15. https://doi.org/10.3171/2014.9.FOCUS14519.

6. Touat M, Idbaih A, Sanson M, Ligon KL. Glioblastoma targeted therapy: updated approaches from recent biological insights. Ann Oncol. 2017;28(7): 1457-72. https://doi.org/10.1093/annonc/mdx106.

7. Jeck WR, Sharpless NE. Detecting and characterizing circular RNAs. Nat Biotechnol. 2014;32(5):453-61. https://doi.org/10.1038/nbt.2890.

8. Chen B, Huang S. Circular RNA: an emerging non-coding RNA as a regulator and biomarker in cancer. Cancer Lett. 2018;418:41-50. https://doi.org/10.101 6/j.canlet.2018.01.011.

9. Kristensen LS, Hansen TB, Venø MT, Kjems J. Circular RNAs in cancer: opportunities and challenges in the field. Oncogene. 2018;37(5):555-65. https://doi.org/10.1038/onc.2017.361.

10. Zhou J, Wang H, Chu J, Huang Q, Li G, Yan Y, et al. Circular RNA hsa_circ_ 0008344 regulates glioblastoma cell proliferation, migration, invasion, and apoptosis. J Clin Lab Anal. 2018;32(7):e22454. https://doi.org/10.1002/jcla.224 54.

11. Wang R, Zhang S, Chen X, Li N, Li J, Jia R, et al. ElF4A3-induced circular RNA MMP9 (circMMP9) acts as a sponge of miR-124 and promotes glioblastoma multiforme cell tumorigenesis. Mol Cancer. 2018;17(1):166. https://doi.org/1 0.1186/s12943-018-0911-0.

12. Cesana M, Daley GQ. Deciphering the rules of ceRNA networks. Proc Natl Acad Sci U S A. 2013;110(18):7112-3. https://doi.org/10.1073/pnas.13 05322110

13. Zhong $Y$, du $Y$, Yang $X$, Mo $Y$, Fan $C$, Xiong F, et al. Circular RNAs function as ceRNAs to regulate and control human cancer progression. Mol Cancer. 2018;17(1):79. https://doi.org/10.1186/s12943-018-0827-8.

14. Chan JJ, Tay Y. Noncoding RNA:RNA Regulatory Networks in Cancer. Int J Mol Sci. 2018;19(5):19-5. https://doi.org/10.3390/ijms19051310.

15. Yu J, Wu SW, Wu WP. A tumor-suppressive microRNA, miRNA-485-5p, inhibits glioma cell proliferation and invasion by down-regulating TPD52L2. Am J Transl Res. 2017:9(7):3336-44.

16. Ou R, Lv J, Zhang Q, Lin F, Zhu L, Huang F, et al. circAMOTL1 motivates AMOTL1 expression to facilitate cervical Cancer growth. Mol Ther Nucleic Acids. 2020;19:50-60. https://doi.org/10.1016/j.omtn.2019.09.022.

17. Qian B, Nag S, Su Y, Voruganti S, Qin JJ, Zhang R, et al. miRNAs in cancer prevention and treatment and as molecular targets for natural product anticancer agents. Curr Cancer Drug Targets. 2013;13(5):519-41. https://doi. org/10.2174/15680096113139990031.

18. Woo HH, Baker T, Laszlo C, Chambers SK. Nucleolin mediates microRNAdirected CSF-1 mRNA deadenylation but increases translation of CSF-1 mRNA. Mol Cell Proteomics. 2013;12(6):1661-77. https://doi.org/10.1074/ mcp.M112.025288.

19. Yang C, Cai J, Wang Q, Tang H, Cao J, Wu L, et al. Epigenetic silencing of miR-130b in ovarian cancer promotes the development of multidrug resistance by targeting colony-stimulating factor 1. Gynecol Oncol. 2012; 124(2):325-34. https://doi.org/10.1016/j.ygyno.2011.10.013.

20. De I, et al. CSF1 overexpression promotes high-grade glioma formation without impacting the polarization status of glioma-associated microglia and macrophages. Cancer Res. 2016;76(9):2552-60. https://doi.org/10.1158/ 0008-5472.CAN-15-2386

21. Stafford JH, Hirai T, Deng L, Chernikova SB, Urata K, West BL, et al. Colony stimulating factor 1 receptor inhibition delays recurrence of glioblastoma after radiation by altering myeloid cell recruitment and polarization. NeuroOncology. 2016;18(6):797-806. https://doi.org/10.1093/neuonc/nov272. 
22. Holdt LM, Kohlmaier A, Teupser D. Molecular functions and specific roles of circRNAs in the cardiovascular system. Noncoding RNA Res. 2018;3(2):75-98. https://doi.org/10.1016/j.ncrna.2018.05.002.

23. Tanguturi SK, Trippa L, Ramkissoon SH, Pelton K, Knoff D, Sandak D, et al. Leveraging molecular datasets for biomarker-based clinical trial design in glioblastoma. Neuro-Oncology. 2017;19(7):908-17. https://doi.org/10.1093/ neuonc/now312.

24. Zhang S, Liao K, Miao Z, Wang Q, Miao Y, Guo Z, et al. CircFOXO3 promotes glioblastoma progression by acting as a competing endogenous RNA for NFAT5. Neuro-Oncology. 2019;21(10):1284-96. https://doi.org/10.1093/ neuonc/noz128.

25. Zhang G, Sun W, Zhu L, Feng Y, Wu L, Li T. Overexpressed circ_0029426 in glioblastoma forecasts unfavorable prognosis and promotes cell progression by sponging miR-197. J Cell Biochem. 2019;120(6):10295-302. https://doi. org/10.1002/jcb.28313.

26. Barbagallo D, Caponnetto A, Brex D, Mirabella F, Barbagallo C, Lauretta G, et al. CircSMARCA5 Regulates VEGFA mRNA Splicing and Angiogenesis in Glioblastoma Multiforme Through the Binding of SRSF1. Cancers (Basel). 2019;11(2):11-2. https://doi.org/10.3390/cancers11020194.

27. Dai X, Chen C, Yang Q, Xue J, Chen X, Sun B, et al. Exosomal circRNA_ 100284 from arsenite-transformed cells, via microRNA-217 regulation of $\mathrm{EZH} 2$, is involved in the malignant transformation of human hepatic cells by accelerating the cell cycle and promoting cell proliferation. Cell Death Dis. 2018;9(5):454. https://doi.org/10.1038/s41419-018-0485-1.

28. Nayak S, Aich M, Kumar A, Sengupta S, Bajad P, Dhapola P, et al. Novel internal regulators and candidate miRNAs within miR-379/miR-656 miRNA cluster can alter cellular phenotype of human glioblastoma. Sci Rep. 2018; 8(1):7673. https://doi.org/10.1038/s41598-018-26000-8.

29. Tu J, Zhao Z, Xu M, Chen M, Weng Q, Ji J. LINC00460 promotes hepatocellular carcinoma development through sponging miR-485-5p to up-regulate PAK1. Biomed Pharmacother. 2019;118:109213. https://doi.org/1 0.1016/j.biopha.2019.109213.

30. Han DL, Wang LL, Zhang GF, Yang WF, Chai J, Lin HM, et al. MiRNA-485-5p, inhibits esophageal cancer cells proliferation and invasion by downregulating O-linked $\mathrm{N}$-acetylglucosamine transferase. Eur Rev Med Pharmacol Sci. 2019;23(7):2809-16. https://doi.org/10.26355/eurrev_201 904_17556.

31. Hsu WC, et al. CSF-1 overexpression predicts poor prognosis in upper tract urothelial carcinomas. Dis Markers. 2019;2019:2724948.

32. Toy EP, Azodi M, Folk NL, Zito CM, Zeiss CJ, Chambers SK. Enhanced ovarian cancer tumorigenesis and metastasis by the macrophage colonystimulating factor. Neoplasia. 2009;11(2):136-44. https://doi.org/10.1593/neo. 81150.

33. Li X, Kong S, Cao Y. miR-1254 inhibits progression of glioma in vivo and in vitro by targeting CSF-1. J Cell Mol Med. 2020;24(5):3128-38. https://doi. org/10.1111/jcmm.14981.

\section{Publisher's Note}

Springer Nature remains neutral with regard to jurisdictional claims in published maps and institutional affiliations.

Ready to submit your research? Choose BMC and benefit from:

- fast, convenient online submission

- thorough peer review by experienced researchers in your field

- rapid publication on acceptance

- support for research data, including large and complex data types

- gold Open Access which fosters wider collaboration and increased citations

- maximum visibility for your research: over $100 \mathrm{M}$ website views per year

At BMC, research is always in progress.

Learn more biomedcentral.com/submissions 